\title{
Physicochemical characterization of spray dried cefixime polymeric nanoparticles using factorial design approach
}

\author{
Pooja Satish Desai, Yogesh Vilas Pore \\ Government College of Pharmacy, Karad, Maharashtra, 415124, India.
}

\begin{tabular}{|c|c|}
\hline ARTICLE INFO & ABSTRACT \\
\hline Article history: & \multirow{6}{*}{$\begin{array}{l}\text { The objective of the present study was to improve the physicochemical properties of poorly water soluble } \\
\text { cefixime by preparing its nanoparticles using a hydrophilic polymer polyvinylpyrrolidone K } 30 \text { (PVP). The } \\
\text { nanoparticles of cefixime were prepared by spray drying technique. A } 3^{2} \text { factorial design approach was } \\
\text { employed for the optimization of nanoparticle batches. The present model demonstrated significance of factors } \\
\text { such as drug to polymer ratio }\left(\mathrm{X}_{1}\right) \text { and feed flow rate }\left(\mathrm{X}_{2}\right) \text { of spray dryer on the production yield }\left(\mathrm{Y}_{1}\right) \text {, particle } \\
\text { size }\left(\mathrm{Y}_{2}\right) \text { and } \% \text { drug release at } 15 \mathrm{~min}\left(\mathrm{Y}_{3}\right) \text {. The pure drug and prepared nanoparticles were characterized by } \\
\text { Fourier transformation infrared spectroscopy (FTIR), Differential scanning calorimetry (DSC), X-ray powder } \\
\text { diffraction (XPRD), Scanning electron microscopy (SEM), particle size, saturation solubility and in vitro drug } \\
\text { release. The results of DSC and XPRD revealed transformation of the crystalline nature of cefixime into an } \\
\text { amorphous form which was supported by SEM. A significant improvement in solubility and dissolution rate was } \\
\text { noticed in case of prepared nanoparticles as compared to pure cefixime. These results suggested that preparation } \\
\text { of polymeric nanoparticles by spray drying technique might be a promising approach for improving the } \\
\text { physicochemical properties of cefixime. }\end{array}$} \\
\hline Received on: 09/01/2016 & \\
\hline Revised on: $10 / 02 / 2016$ & \\
\hline Accepted on: 17/03/2016 & \\
\hline Available online: $30 / 04 / 2016$ & \\
\hline $\begin{array}{l}\text { Key words: } \\
\text { Cefixime; factorial design; } \\
\text { spray drying; nanoparticles; } \\
\text { polymeric; physicochemical } \\
\text { characterization. }\end{array}$ & \\
\hline
\end{tabular}

\section{INTRODUCTION}

It is well known that physicochemical properties such as solubility, dissolution and permeability are the major governing factors for the rate and extent of absorption and bioavailability of drug molecules (Amidon et al., 1995). In the present scenario, oral drug delivery is continuously looking into newer avenues as $70 \%$ of new drug candidates have poor aqueous solubility (Kawabata et al., 2011). The drugs with low aqueous solubility usually show dissolution rate-limited absorption (Proudfoot, 1991) which affects their overall therapeutic outcome. Therefore, dissolution profile of poorly aqueous soluble drugs has been recognized as a key parameter in the drug development process (Khadka et al., 2014). Thus improving the physicochemical properties of poorly soluble

\footnotetext{
* Corresponding Author

Yogesh Vilas Pore, Government College of Pharmacy, Karad, Maharashtra, 415124, India. Email: dryogeshpore[at]rediffmail.com
}

drugs and enhancing their bioavailability has become a challenging task to pharmaceutical scientists. Recently, major research efforts have been focused on the development of particulate drug delivery systems like microparticles, nanoparticles, dendrimers, polymeric micelles and solid lipid nanoparticles, which have become promising approaches to alter the physicochemical properties of drugs without affecting their pharmacological action (Alanazi et al., 2007; Sahoo et al., 2011; Dixit et al., 2015). Polymeric nanoparticles are sub-nanosized colloidal structure in which the drug is dissolved, entrapped, encapsulated or attached to a nanoparticle matrix.

Particle size reduction to the nanometer range can be achieved by using different techniques. This technique has been extensively described relative to particle size reduction efficiently such as solvent evaporation method, nanoprecipitation method, ion gelation/coacervation technique, spray drying method, and supercritical antisolvent technique to improve drug solubility (Hecq et al., 2005; Khadka et al., 2014; Yadav et al., 2012; Singh et al., 2013). 
Among the formulation strategies, spray drying method is used for preparation of nanoparticles as it is a single stage continuous process and can be easily adapted to industrial scale. Also, spray dried particles have approximately spherical shape, free flowing, narrow particle size distribution and high drug loading efficiency (Kristmundsdottir et al., 1996; Sahoo et al., 2011).

Cefixime (Fig. 1) is an oral third generation cephalosporin antibiotic used in the treatment of uncomplicated urinary tract infection, pharyngitis, acute bronchitis and uncomplicated gonorrhea. It has an oral bioavailability of about $40 \%$ - 50\% and plasma half-life 3-4 hrs. According to the Biopharmaceutical Classification System (BCS), cefixime belongs to BCS class IV drugs, which are often characterized by low permeability and low solubility, resulting in its limited and variable oral bioavailability (Jadhav et al., 2013).

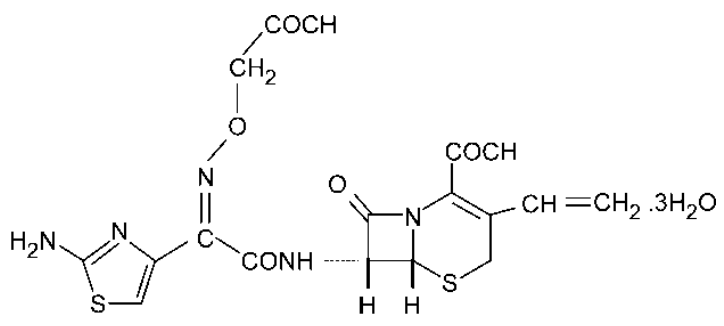

Fig. 1: Chemical structure of cefixime.

Various studies have been reported on different cefixime formulations such as inclusion complex formation, microparticles, microspheres, nanoparticles (by modified precipitation method) and nanocrystals (Jadhav et al.,2013; Raval et al., 2012; Murtaza et al., 2012; Sonam et al., 2014; Hussein and Mahmood 2014) to evaluate the solubility and release kinetics of cefixime.

As the absorption / bioavailability of BCS Class IV drugs is both dissolution rate and permeability limited, these drugs are poor candidates for drug development since mere improvement in solubility and dissolution will not suffice the bioavailability enhancement purpose. However, because of only permeability issues, these candidates may not be overlooked from the formulation point of view. Therefore, certain other approaches in addition to those being used for BCS Class II drugs should be employed to address the bioavailability problems of such candidates (Khadka et al., 2014). In this article, particle size reduction to nano range in the form of polymeric nanoparticles has been employed to improve the solubility and dissolution of cefixime. The formation of polymeric material and reduction to the nanometer range would be expected to be effective techniques for the improvement of desired transport properties via increasing solubility of drug candidate (Rao et al., 2011). Further, the factorial design approach was used to optimize the physicchemical properties of polymeric nanoparticles of cefixime.

The aim of this research work was to prepare polymeric nanoparticles of cefixime by spray drying technique using hydrophilic carrier polyvinylpyrrolidone K30 (PVP) in order to improve its solubility and dissolution rate. The formulation was optimized by using $3^{2}$ factorial design approach. It provides a method to develop an empirical model equation to characterize the response as a function of the different independent variables (Hamed and Sakr, 2001; Padamwar and Pokharkar, 2006; Rhee et al., 2008). The optimized formulation batches were further characterized by Fourier transformation infrared spectroscopy (FTIR), X-ray powder diffractometry (XPRD), Scanning electron microscopy (SEM), Differential scanning calorimetry (DSC), particle size analysis, saturation solubility and in vitro dissolution studies in $0.01 \mathrm{~N} \mathrm{HCL}$.

\section{MATERIALS AND METHODS}

\section{Materials}

Cefixime was obtained as a gift sample from Okasa Pharma Ltd., Satara, India. PVP was gifted by Indoco Remedies, Mumbai, India. Analytical grade chemicals and double distilled water were used for all experimental procedures.

\section{Preparation of cefixime nanoparticles by spray drying method}

Drug loaded polymeric nanoparticles were prepared in 1:1, 1:2 and 1:3 drug: PVP ratios. The drug and polymer were dissolved in methanol and the solution was stirred on a magnetic stirrer at room temperature $\left(25 \pm 2{ }^{\circ} \mathrm{C}\right)$ to obtain the feed solution. The feed solution was spray dried using the Lab spray dryer (SPDD-111 Techno Search Instruments, Thane, India) using a standard $0.7 \mathrm{~mm}$ nozzle under the following set of conditions: Inlet temperature $65{ }^{\circ} \mathrm{C}$, outlet temperature $45^{\circ} \mathrm{C}$, aspirator speed 40 $\mathrm{mBar}$, feed flow rate 3,4 and $5 \mathrm{~mL} / \mathrm{min}$. The spray dried particles were collected and stored in desiccators until subjected for further analysis.

\section{Effect of variables}

To examine the effect of variables on nanoparticle performance and characteristics, different batches were prepared using $3^{2}$ factorial design. In this design, 2 factors were evaluated at 3 levels and experimental trials were performed in all 9 possible combinations. The 2 independent variables investigated were the amount of drug to polymer ratio $\left(\mathrm{X}_{1}\right)$ and feed flow rate $\left(\mathrm{X}_{2}\right)$ of spray dryer. The production yield $\left(\mathrm{Y}_{1}\right)$, particle size $\left(\mathrm{Y}_{2}\right)$ and percentage drug release at $15 \min \left(\mathrm{Y}_{3}\right)$ were chosen as dependent variables. The levels for formulation as well as process variables were determined from preliminary trials.

\section{Percentage production yield}

The percentage of production yield of nanoparticle was calculated using the weight of the final product after drying with respect to the initial total weight of the drug and polymer used for the preparation of nanoparticles.

\section{Determination of drug content}

Drug content was determined by dissolving prepared nanoparticles equivalent to $5 \mathrm{mg}$ of the drug in $5 \mathrm{ml}$ of methanol and then the volume was adjusted up to $50 \mathrm{ml}$ with distilled water. 
The solution was sonicated for $10 \mathrm{~min}$ and filtered through the Whatmann filter paper 41 . The aliquots were suitably diluted and absorbance was measured at $288 \mathrm{~nm}$ by using double beam UV-visible spectrophotometer (Shimadzu 1800, Japan).

\section{Saturation solubility studies}

Saturation solubility studies were performed in distilled water as follows: an excess amount of pure drug and / or nanoparticles were added to $10 \mathrm{ml}$ of distilled water taken in screw cap tube and shaken for $24 \mathrm{~h}$ in a rotary flask shaker at a room temperature $\left(25 \pm 0.5{ }^{\circ} \mathrm{C}\right)$. Appropriate aliquots were then withdrawn and filtered through Whatmann filter paper 41 . The filtrate was suitably diluted with distilled water and analyzed by UV-visible spectrophotometer (Shimadzu 1800, Japan) at $288 \mathrm{~nm}$.

\section{Particle size analysis}

Determination of the mean average particle size of cefixime nanoparticles was carried out by using Malvern Mastersizer 2000 SM, (Malvern Instruments Corp., Worcestershire, U.K.) based on laser diffraction technique. Analysis was carried out at $30 \pm 2{ }^{\circ} \mathrm{C}$ temperature keeping the angle of detection $90^{\circ}$. The particle size distribution is given by $\mathrm{d}(0.9), \mathrm{d}(0.5)$ and $(0.1)$ which is the particle size diameters determined at $90^{\text {th }}, 50^{\text {th }}$, and the $10^{\text {th }}$ percentile of particle undersized, respectively. The width of the size distribution was indicated by span value.

\section{Zeta potential determination}

The surface charge was determined by measuring the electrophoretic mobility of nanoparticles by using Nanoparticle size analyzer (Horbia SZ-100, Kyoto, Japan). The zeta potential was measured after dilution of samples with distilled water at room temperature.

\section{In vitro dissolution studies}

The dissolution studies were carried out using USP dissolution test apparatus (DS 8000 LABINDIA, LABINDIA Analytical Instruments, Pvt. Ltd. Mumbai, India), according to USP type II. Samples were placed in a dissolution vessel containing $900 \mathrm{ml}$ of $0.01 \mathrm{~N} \mathrm{HCL}$ at $50 \mathrm{rpm}$, maintained at $37 \pm 0.5$ ${ }^{\circ} \mathrm{C}$ (Jadhav et al., 2013). $100 \mathrm{mg}$ of drug or its equivalent amount of nanoparticles were added to dissolution medium. Five $\mathrm{ml}$ of samples was withdrawn by auto sampler at previously programmed time intervals of $5,10,15,20,30,45$ and $60 \mathrm{~min}$ maintaining the sink condition. The samples were immediately filtered through Whatmann filter paper 41; suitably diluted and analyzed spectrophotometrically (Shimadzu 1800, Japan) at 284.5 $\mathrm{nm}$.

\section{Response surface analysis}

Response surface analysis was carried out using STATEASE (Design Expert, Version 9.0.4.1) software which was used for $3^{2}$ factorial design. A linear regression model equation was employed for fitting the surface in the following term.

$$
\mathrm{Y}=\mathrm{b}_{0}+\mathrm{b}_{1} \mathrm{X}_{1}+\mathrm{b}_{2} \mathrm{X}_{2}+\mathrm{b}_{12} \mathrm{X}_{1} \mathrm{X}_{2}+\mathrm{b}_{11} \mathrm{X}_{1}^{2}+\mathrm{b}_{22} \mathrm{X}_{2}^{2}
$$

Where $\mathrm{Y}$ is the predicted response, $\mathrm{b}_{0}$ is the arithmetic mean of 9 runs. The main effects $\left(X_{1}\right.$ and $\left.X_{2}\right)$ represent the average result of changing one factor at a time from its low value to its high value. The interaction $\left(\mathrm{X}_{1}, \mathrm{X}_{2}\right)$ shows how the percentage production yield, particle size and percentage drug release at 15 min value changes when two factors are simultaneously changed, and the exponential terms $\left(\mathrm{X}_{1}{ }^{2}\right.$ and $\left.\mathrm{X}_{2}{ }^{2}\right)$ represents curvature. The coefficients corresponding linear effects $\left(b_{1}\right.$ and $\left.b_{2}\right)$, interaction $\left(b_{12}\right)$ and the quadratic effects $\left(b_{11} b_{22}\right)$ were determined from the results of the experiment (Shinde et al., 2010). Data were analyzed statistically and regression coefficients were calculated. The optimized batch was determined by consideration of determination coefficients, adjusted determination coefficients which indicates whether of independent variables have significance on dependent variables or not. The surface response plots were also included to provide ideal explanation for the significance of the model.

\section{Differential scanning calorimetry (DSC)}

Thermal properties of pure drug, polymer and an optimized batch of nanoparticles were analyzed by using differential scanning calorimetry (Mettler DSC 823E, Mettler Toledo, Switzerland). An empty aluminum pan was used to calibrate the DSC temperature and enthalpy scale. The samples of (2-5 mg) were hermetically sealed in aluminum crucibles and heated at a constant rate of $10^{\circ} \mathrm{C} / \mathrm{min}$ over a temperature range of 0 to $300^{\circ} \mathrm{C}$. An inert atmosphere was maintained by purging nitrogen gas at a flow rate of $50 \mathrm{~mL} / \mathrm{min}$.

\section{X-ray powder diffractometry (XRPD)}

X-ray diffraction analysis of drug, polymer and an optimized batch of nanoparticles was carried out using $\mathrm{X}$ - ray diffractometer (PW 1729, Philips, The Netherlands). The samples were irradiated with monochromatized $\mathrm{Cu}-\mathrm{K}[\alpha]$ radiation $\left(1.542 \mathrm{~A}^{\circ}\right)$ and analyzed between $5-90^{\circ}(2 \theta)$. The voltage and current used were $30 \mathrm{kV}$ and $30 \mathrm{~mA}$ respectively. The range was $5 \times 10^{3} \mathrm{CPS}$ and the chart speed was kept at $10 \mathrm{~mm} / 2 \theta$.

\section{Fourier transformation infrared spectroscopy (FTIR)}

The infrared spectra of cefixime, polymer and an optimized batch of nanoparticles were obtained using FTIR spectrophotometer (Agilent Cary 630 ATR Model, Agilent Technologies, CA, U.S.A). About 3-4 mg of sample was directly placed on the stage of the spectrometer and scanned over the range of 4000 to $500 \mathrm{~cm}^{-1}$ with the resolution of $4 \mathrm{~cm}^{-1}$.

\section{Scanning electron microscopy (SEM)}

The surface morphology of the drug and optimized batch of nanoparticles were evaluated by scanning electron microscope (JEOL, JSM-6360, Tokyo, Japan) operated at an acceleration voltage of $20 \mathrm{kV}$ and microphotographs were obtained at X2000, X5000 and X10000 magnifications. 


\section{RESULTS AND DISCUSSION}

\section{Percentage production yield}

Percentage production yield of prepared nanoparticles was obtained in the range of $29.48 \%$ to $47.17 \%$. The minimum production yield $(29.48 \%)$ was obtained for the batch F7 which was spray dried at a flow rate of $5 \mathrm{ml} / \mathrm{min}$ containing 1:1 drug to polymer ratio whereas the maximum production yield $(47.17 \%)$ was obtained for the batch $\mathrm{F} 3$ which was spray dried at a flow rate of $3 \mathrm{ml} / \mathrm{min}$ containing 1:3 drug to polymer ratio. The percentage production yield for all other batches $(\mathrm{F} 1, \mathrm{~F} 2, \mathrm{~F} 4, \mathrm{~F} 5, \mathrm{~F} 6, \mathrm{~F} 8$ and F9) was obtained in the range of $32.75 \%$ to $43.88 \%$ (Tale 2 ). The effect of variables on the production yield of various batches has been discussed in response surface analysis section.

\section{Determination of drug content}

Percentage drug content of the spray dried nanoparticles was found in the range of $95.15 \pm 0.48 \mathrm{w} / \mathrm{w}$ to $99.43 \pm 0.38 \mathrm{w} / \mathrm{w}$.

\section{Saturation solubility studies}

The results of saturation solubility studies of all samples are shown in Table 1. It was evident that solubility of all batches of the nanoparticles increased significantly $(p<0.05)$ as compared to pure drug alone. The physical characterization studies confirmed the presence of high energy and highly disordered amorphous state of polymeric drug nanoparticles, resulting in enhanced drug solubility (Lepek et al., 2013). In addition to that, solubility enhancement of nanoparticles could be due to an improved wettability of drug particles and localized solubilization by the hydrophilic polymer with simultaneous reduction in particle size (Bhosale et al., 2012; Jamadar et al., 2014).

Table. 1: Saturation solubility of pure cefixime and nanoparticle formulations

\begin{tabular}{|c|c|}
\hline Batch Code & 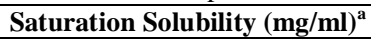 \\
\hline Pure cefixime & $0.57 \pm 0.01$ \\
\hline $\mathrm{F} 1$ & $3.52 \pm 0.08^{\mathrm{b}}$ \\
\hline $\mathrm{F} 2$ & $4.96 \pm 0.11^{\mathrm{b}}$ \\
\hline F3 & $5.95 \pm 0.12^{\mathrm{b}}$ \\
\hline $\mathrm{F} 4$ & $3.04 \pm 0.09^{\mathrm{b}}$ \\
\hline F5 & $4.41 \pm 0.12^{\mathrm{b}}$ \\
\hline F6 & $5.43 \pm 0.13^{b}$ \\
\hline F7 & $2.67 \pm 0.11^{\mathrm{b}}$ \\
\hline F8 & $3.66 \pm 0.13^{\mathrm{b}}$ \\
\hline F9 & $4.83 \pm 0.09^{\mathrm{b}}$ \\
\hline
\end{tabular}

${ }^{\mathrm{a}}$ Mean \pm S.D. $(\mathrm{n}=3)$ : S.D. standard deviation; ${ }^{\mathrm{b}}$ Significant differences compared to pure cefixime $(p<0.05)$

\section{Particle size and zeta potential determination}

The results of particle size analysis are shown in Table 2. The particle size distribution of pure cefixime, an optimized batch of cefixime nanoparticles (F3) and zeta potential of the optimized batch (F3) are shown in Figure 2 and 3 respectively. The average particle size of pure cefixime powder was $68519 \mathrm{~nm}$ with a span value of 25.205 indicating very broad size distribution of a drug. The average particle size of prepared nanoparticles (F1 to F9) was found to be in the range of 107.9 to $343.1 \mathrm{~nm}$ with a span value in the range of 0.029 to 1.570 . Batch F3 was optimized on the basis of small particle size and more percentage drug release with high production yield. The particle size of F3 batch was found to be 197 $\mathrm{nm}$ with span value of 1.119 indicating quite narrow and uniform size distribution as compared to pure drug particles (Shah et al., 2011).

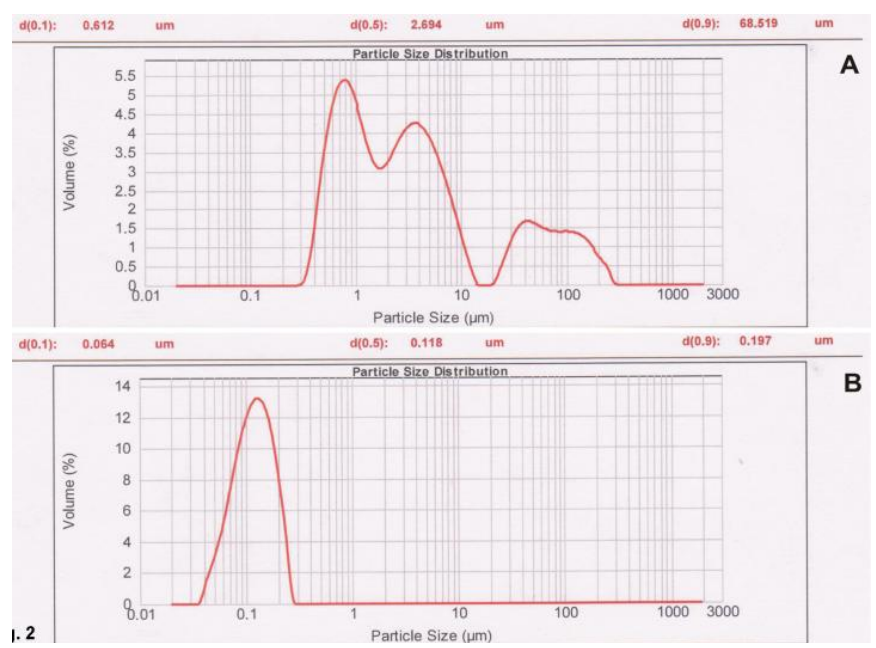

Fig. 2: The particle size distribution of pure cefixime (A) and optimized batch of cefixime nanoparticles (F3) (B).

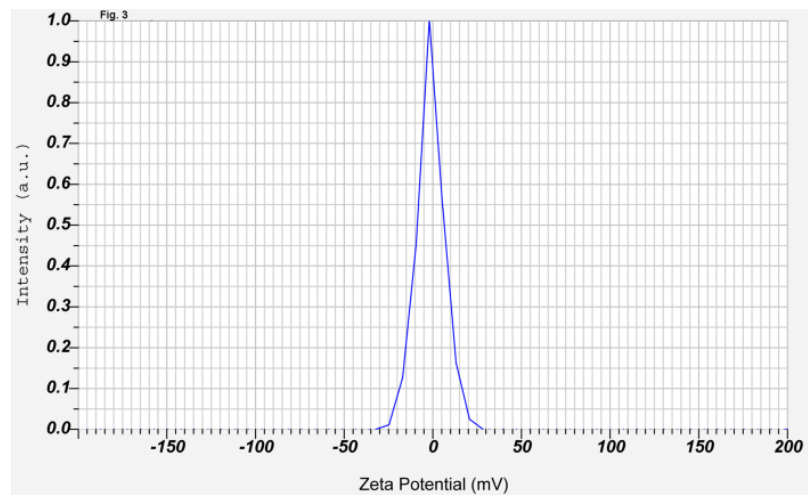

Fig. 3: Zeta potential of optimized batch (F3).

Zeta potential of optimized batch $\mathrm{F} 3$, measured in distilled water was found to be $-1.3 \mathrm{mv}$. The criteria for selection of solvent is that the particles do not dissolve, swell and /or change chemically in liquid.

The water may be used as a solvent if the powder solubility is $<5 \mathrm{~g}$ powder in $1 \mathrm{~kg}$ of water (http://www.gammadata.se/assets/PowderDispersions.pdf). The solubility of optimized batch F3 nanoparticles was found to be $5.95 \pm 0.12 \mathrm{mg} / \mathrm{ml}$ in distilled water (Table 1 ). The refractive index, conductivity, dielectric constant, $\mathrm{pH}$ and viscosity of the liquid including wetting of the particles by the liquid are the other factors those should be considered while selecting a solvent for analysis (http://www3.nd.edu/ rroeder/ame60647/slides/zeta.pdf). The use of liquid provides a better approach for analyzing individual vs. agglomerated particles (http://www.gammadata.se/assets/PowderDispersions.pdf). The high potential values were necessary to be achieved to ensure a high energy barrier and impart a good stability to the formulation. 
Table 2: Experimental design and parameters for $3^{2}$ full factorial design of F1 to F9 batches.

\begin{tabular}{|c|c|c|c|c|c|c|c|}
\hline \multirow[t]{2}{*}{ Batch Code } & \multicolumn{2}{|c|}{ Coded Value } & \multicolumn{2}{|c|}{ Actual Value } & \multicolumn{3}{|c|}{ Dependent Variables } \\
\hline & $\mathbf{X}_{1}$ & $\mathbf{X}_{2}$ & $\mathbf{X}_{1}$ & $\mathbf{X}_{2}$ & $\%$ Production Yield $\left(\mathrm{Y}_{1}\right)$ & Particle size $(\mathrm{nm})\left(\mathrm{Y}_{2}\right)$ & $\%$ Drug release at $15 \min ^{\mathrm{a}}\left(\mathrm{Y}_{3}\right)$ \\
\hline Cefixime & ---- & ---- & ---- & ---- & ---- & 68519 & $13.24 \pm 0.76$ \\
\hline $\mathrm{F} 1$ & -1 & -1 & $1: 1$ & 3 & 34.63 & 107.9 & $39.73 \pm 0.53$ \\
\hline F2 & 0 & -1 & $1: 2$ & 3 & 43.88 & 140.9 & $49.67 \pm 0.45$ \\
\hline F3 & 1 & -1 & $1: 3$ & 3 & 47.17 & 197.0 & $99.21 \pm 0.44$ \\
\hline $\mathrm{F} 4$ & -1 & 0 & $1: 1$ & 4 & 32.75 & 214.4 & $45.96 \pm 0.79$ \\
\hline F5 & 0 & 0 & $1: 2$ & 4 & 40.53 & 263.4 & $47.02 \pm 0.62$ \\
\hline F6 & 1 & 0 & $1: 3$ & 4 & 44.76 & 329.0 & $95.73 \pm 0.46$ \\
\hline F7 & -1 & 1 & $1: 1$ & 5 & 29.48 & 216.9 & $37.55 \pm 0.62$ \\
\hline F8 & 0 & 1 & $1: 2$ & 5 & 39.76 & 323.6 & $41.31 \pm 0.71$ \\
\hline F9 & 1 & 1 & $1: 3$ & 5 & 42.10 & 343.1 & $85.89 \pm 0.79$ \\
\hline
\end{tabular}

$\mathrm{X}_{1}$ : drug: polymer ratio (mg: $\left.\mathrm{mg}\right), \mathrm{X}_{2}$ : feed flow rate $(\mathrm{ml} / \mathrm{min}) ;{ }^{\mathrm{a}}$ Mean \pm S.D. $(\mathrm{n}=3)$ : S.D. standard deviation.

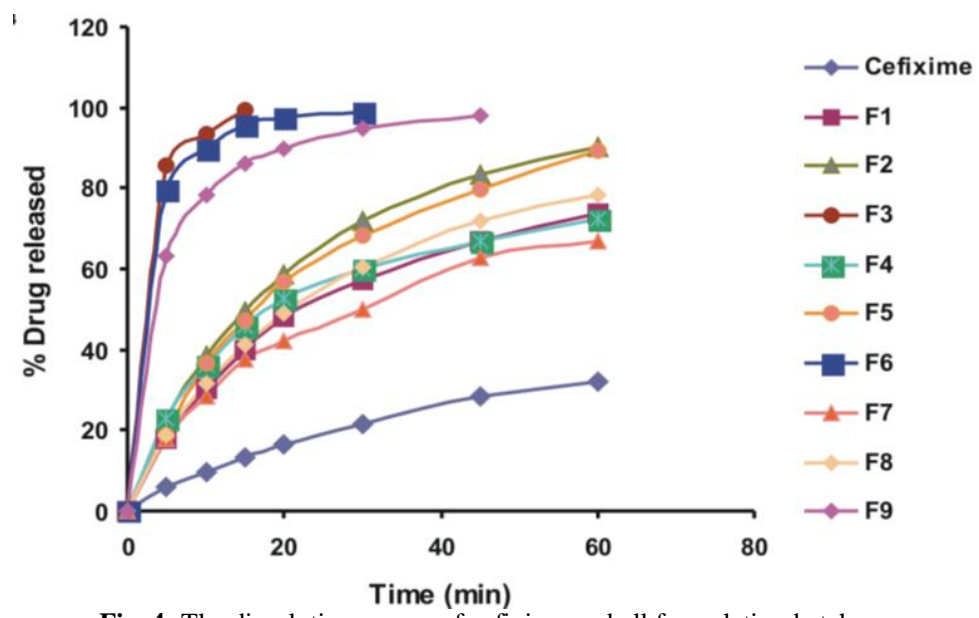

Fig. 4: The dissolution curves of cefixime and all formulation batches.

\section{In vitro dissolution studies}

The dissolution profile of pure cefixime and all batches of nanoparticles (F1 to F9) are shown in Table 2 and Figure 4. The release rate profiles were expressed as the cumulative percentage drug release $(\%)$ versus time (min). From the data the onset of dissolution of pure cefixime was poor initially (13.24 $\pm 0.76 \%$ within $15 \mathrm{~min})$ and also during entire dissolution period $(32.153 \pm$ $0.55 \%$ within $60 \mathrm{~min}$ ). The dissolution rate of cefixime nanoparticles was affected by polymer concentration and flow rate of spray drying method. The dissolution rates of cefixime nanoparticles were significantly enhanced as compared to pure drug $(p<0.001)$. The spray drying process itself was responsible for increased dissolution. It has been shown to reduce aggregation tendencies of particles leading to increase in dissolution rate. Further, use of hydrophilic carrier which was responsible for creating hydrophilic layer around the drug particles led to improved wettability and dispensability of particles and there by increased dissolution rate (Jamadar et al., 2014; Kornblum et al.,1971; Kornblum et al.,1971). The prevention of aggregation and agglomeration of individual drug particles exhibiting a high solid-liquid surface tension, creation of a microenvironment in which the drug solubility is high and stabilization of an amorphous state due to antiplasticizing effect of hydrophilic polymer might have also contributed for an improved dissolution performance (Jondhale et al., 2012). Another reason for improvement in the dissolution profile of nanoparticles might be attributed to altered size, morphology and conversion of crystalline drug to amorphous form as confirmed by DSC and XPRD studies (Jamadar et al.,2013). According to Noyes-Whitney equation the dissolution rate was directly proportional to its surface area exposed to the dissolution medium (Noyes and Whitney 1897). The increased dissolution of drug nanoparticles could thus be mainly attributed to their increased surface area in comparison with pure drug (Bhosale et al., 2012).

\section{Response surface analysis}

Experimental design with coded level of variable and their actual values including the effects of the different combination of concentration of drug and polymer ratio $\left(\mathrm{X}_{1}\right)$ and feed flow rate $\left(\mathrm{X}_{2}\right)$ on the responses such as a production yield $\left(\mathrm{Y}_{1}\right)$, particle size $\left(\mathrm{Y}_{2}\right)$ and percentage drug release at $15 \mathrm{~min}\left(\mathrm{Y}_{3}\right)$ are illustrated in Table 2. The response surface plots are shown in Figure 5.

The application of responses surface methodology yielded the following regression equations, which suggested an empirical relationship between the values of responses and independent variables in coded unit. The value of the dependent variables of cefixime nanoparticles formulations is described in Table 2 .

These values are necessary to get polynomial equations for the respective dependent variable. The response surface data were fitted in above equation 1 and the fitting models for production yield, particle size and percentage in vitro drug release at 15 min were given in equation 2, 3, 4 respectively. 
$Y_{1}=41.29+6.19 \mathrm{X}_{1}-2.39 \mathrm{X}_{2}+0.020 \mathrm{X}_{1} \mathrm{X}_{2}-2.91 \mathrm{X}_{1}^{2}+0.16 \mathrm{X}_{2}^{2} \ldots . .(2)$
$\mathrm{Y}_{2}=237.36+54.98 \mathrm{X}_{1}+72.97 \mathrm{X}_{2} \ldots(3)$
$\mathrm{Y}_{3}=48.68+26.27 \mathrm{X}_{1}-3.98 \mathrm{X}_{2}-2.78 \mathrm{X}_{1} \mathrm{X}_{2}+21.34 \mathrm{X}_{1}^{2}-4.01 \mathrm{X}_{2}^{2} \ldots .(4)$
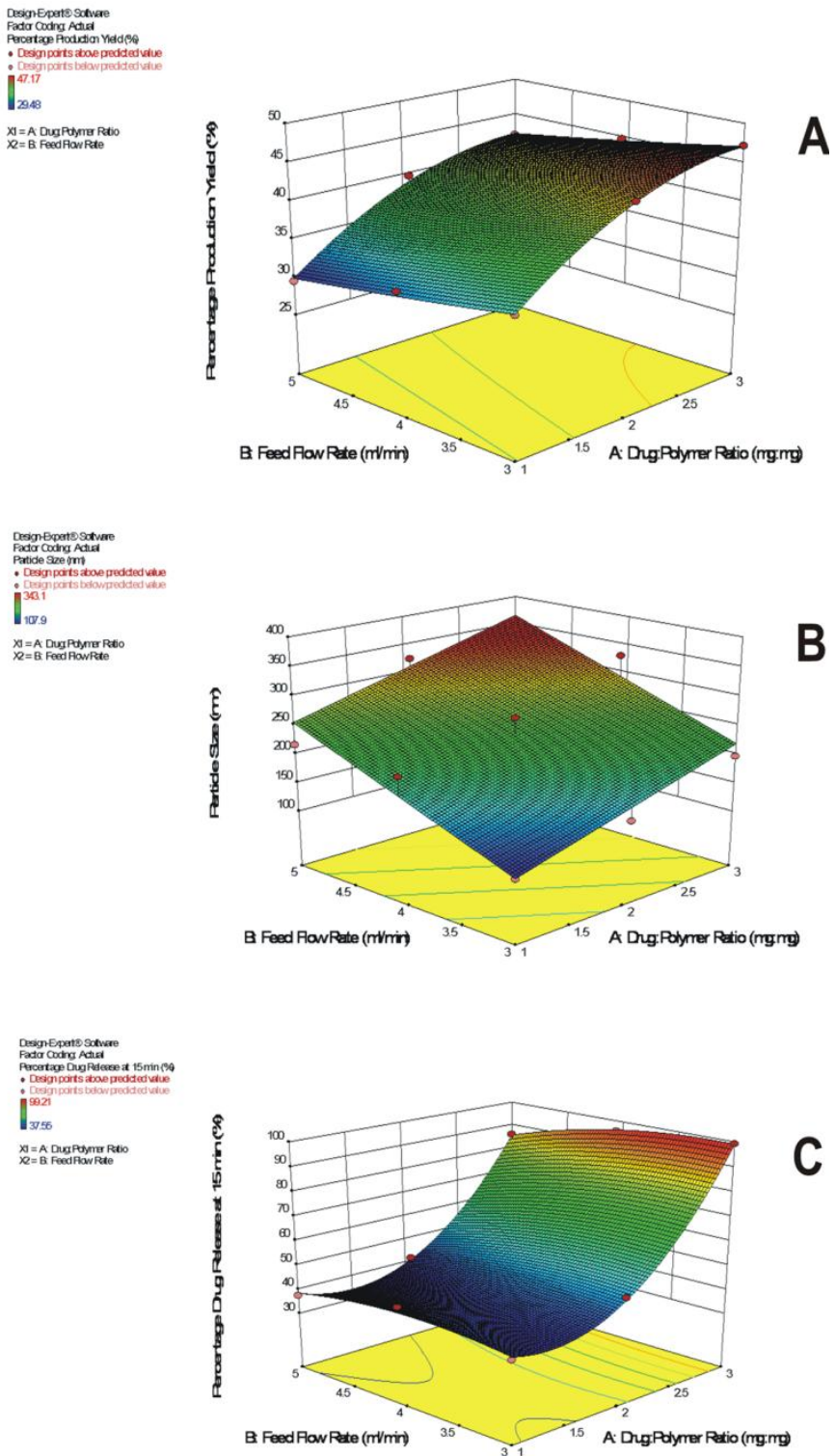

Fig. 5: Response surface plots of effect of variables on $\%$ production yield (A), particle size (B) and \% drug release at $15 \mathrm{~min}(\mathrm{C})$.

The equation 2 and surface response plot (Fig 5A) indicated the positive effect of the drug to polymer ratio on the production yield. With increase in the drug to polymer ratio, the production yield also increased which might be due to increased throughput of the polymer slurry and rapid evaporation of the solvent. However, the negative effect of feed flow rate on the percentage production yield was observed. The production yield was decreased with an increase in the feed flow rate attributed to incomplete atomization and drying, resulting in the deposition of a large amount of particles on the wall of the desiccating chamber and the cyclone separator (Motlekar et al., 2008). The effect of the drug to polymer ratio and feed flow rate on the particle size was obtained from equation 3 as displayed in surface response plot (Fig
5B). The positive effect of the drug to polymer ratio might be correlated to increasing the viscosity of feed solution which interfered with the interaction between dispersed phase and dispersion medium affecting the size distribution of particles (Nagda et al., 2009). The positive effect of feed flow rate on the particle size was observed. Due to higher feed flow rate the atomization air might not be able to penetrate into the stream of liquid. This resulted in incomplete atomization leading to formation of a wide particle size distribution. When considering the response in term of percentage drug release at $15 \mathrm{~min}$, the equation 4 and surface response plot (Fig 5C) indicated positive and negative effects of the drug to polymer ratio and feed flow rate on the percentage drug release at $15 \mathrm{~min}$, respectively. The dissolution rate of the nanoparticles increased with an increase in drug to polymer ratio due to molecular dispersion of the drug in high PVP matrix resulting in a glassy solution as shown in thermograms (Homayouni et al.,2014). Further, as the feed flow rate was decreased, the dissolution rate was increased. It was clear that due to higher pumping rate the larger volume of nebulized solutions was required to be dried during spray drying process. The heated air might not be rapidly transferring the liquid droplets into solid nanoparticles leading to the formation of larger and irregular particles which were not properly and immediately dried (Wan et al.,1991; Shinde et al., 2010). Consequently, decrease in the feed flow rate was favorable to reduce the particle size (equation 3 ) resulting in an improved drug release.

\section{Statistical analysis}

As shown in Table 3, the observed "F- value" was higher than 1.0 which inferred that there was significant variation among group means than expected value. The models with such values of $p<0.001$ were significant by rejection of null hypothesis. The correlation coefficient $\left(\mathrm{R}^{2}\right)$ for response as production yield, particle size and percentage drug release at $15 \mathrm{~min}$ indicated a high significance and good fit of the models. Adequate precision measures the signal to noise ratio.

Table 3: Summary of regression analysis results for measured responses with their coefficients.

\begin{tabular}{lccc}
\hline Coefficients & $\begin{array}{c}\text { \% Production } \\
\text { Yield }\end{array}$ & $\begin{array}{c}\text { Particle Size } \\
(\mathbf{n m})\end{array}$ & $\begin{array}{c}\text { \% drug release } \\
\text { at 15 min }\end{array}$ \\
\hline $\mathrm{b}_{0}$ & 41.29 & 237.36 & 48.68 \\
$\mathrm{~b}_{1}$ & +6.19 & +54.98 & +26.27 \\
$\mathrm{~b}_{2}$ & -2.39 & +72.92 & -3.98 \\
$\mathrm{~b}_{11}$ & -2.91 & --- & +21.34 \\
$\mathrm{~b}_{22}$ & +0.16 & --- & -4.01 \\
$\mathrm{~b}_{12}$ & +0.020 & --- & -2.78 \\
$\mathrm{R}^{2}$ & 0.9939 & 0.8769 & 0.9977 \\
$\mathrm{P}$ & 0.0016 & 0.0019 & 0.0004 \\
$\mathrm{~F}$ value & 98.23 & 21.37 & 260.72 \\
\hline
\end{tabular}

A ratio greater than 4 is desirable, but in this model ratio was observed to be 27.77 for response of production yield, whereas 13.73 and 37.06 for particle size and percentage drug release at $15 \mathrm{~min}$ respectively, indicating an adequate model. By consideration of values $\mathrm{F}, p$, correlation coefficient $\left(\mathrm{R}^{2}\right)$ and adequate precision, it could be concluded that a significant 
synergistic interaction between all two independent variables at $p$ $<0.001$ was observed for $3^{2}$ factorial design model as the result showed that concentration of drug to polymer ratio and feed flow rate had a significant effect on production yield, particle size and percentage drug release at $15 \mathrm{~min}$ of nanoparticle formulations. As a result of $3^{2}$ factorial design, for development of the desired finished product of cefixime nanoparticles, accepted range for each independent variables were as: concentration of drug to polymer ratio $(1: 1-1: 3)$ and feed flow rate $(3-5 \mathrm{ml} / \mathrm{min})$.

\section{Differential scanning calorimetry}

DSC thermogram of the pure cefixime powder, PVP, and an optimized batch of nanoparticles F3 are shown in Figure 6. DSC thermogram of pure cefixime showed a glass transition $\left(\mathrm{T}_{\mathrm{g}}\right)$ at $92.11{ }^{\circ} \mathrm{C}$ and also showed a broad endothermic peak at $125.78{ }^{\circ} \mathrm{C}$. It indicated that the pure cefixime might be in the metastable amorphous form (Jadhav et al., 2013).

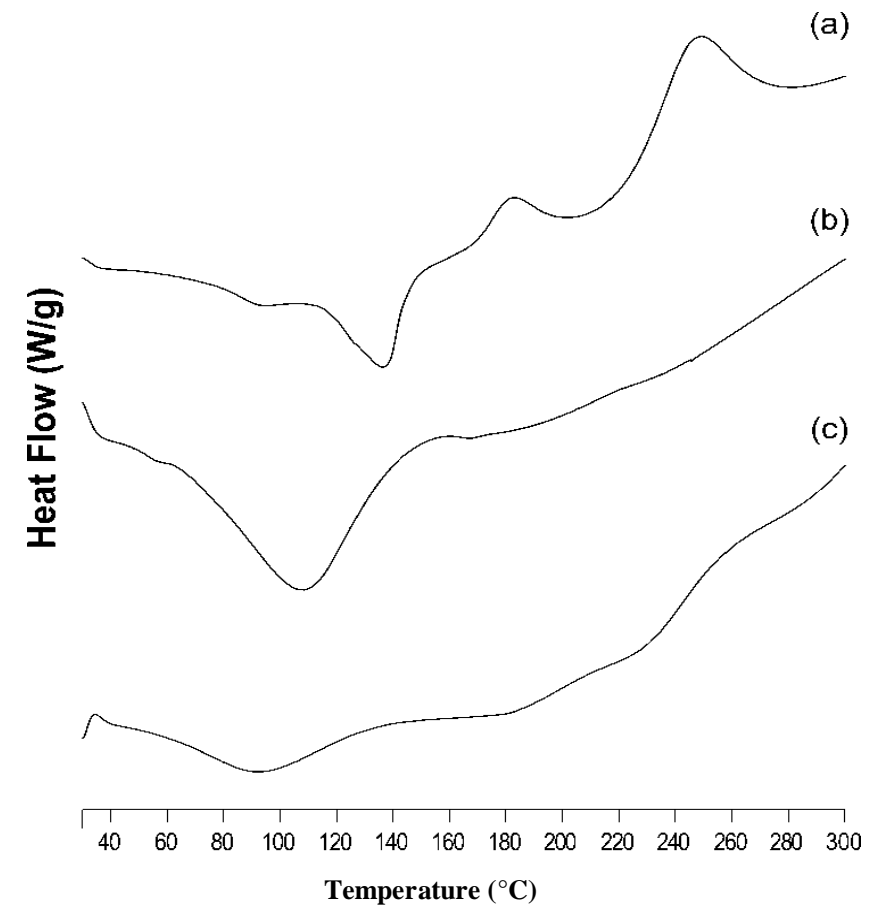

Fig. 6: DSC thermograms of pure cefixime (a), PVP (b) and optimized batch F3 (c).

PVP exhibited a broad endothermic peak at the $107.06{ }^{\circ} \mathrm{C}$ corresponding to loss of water with an absence of melting endothermic peak indicating its amorphous nature (Pandya et al., 2009, Bhosale et al., 2012). DSC thermogram of nanoparticles of the optimized batch (F3) displayed broad endothermic peak at the $92.42{ }^{\circ} \mathrm{C}$ as a result of loss of water. No other noticeable peak was observed, indicating a strong interaction between drug and the carrier. It revealed that cefixime was molecularly dispersed inside the nanoparticles (Tran et al., 2013). As in the spray drying process the spontaneous removal of solvent caused drug molecules to lose their mobility and further the molecules got entrapped in polymer without any crystal structure (Roni et al., 2011).

\section{X-ray powder diffractiometry (XPRD)}

The XPRD pattern of pure cefixime, PVP, and an optimized batch of nanoparticles F3 are shown in Figure 7. The XRPD of pure cefixime exhibited characteristic intensity diffraction peaks between $9.05^{\circ}$ to $26.45^{\circ} 2 \theta$ with some diffused peaks indicating its metastable amorphous form as obtained in DSC thermogram (Jadhav et al., 2013). The XPRD of PVP showed complete absence of any diffraction peaks, which was characteristic of an amorphous nature of polymer (Jondhale et al., 2012). Comparing diffraction patterns of pure cefixime with prepared nanoparticles, the XPRD diffractogram of nanoparticles of optimized batch F3 did not show any sharp intense peak; rather it showed halo pattern confirming that the drug was converted into an amorphous state which was in good agreement with the results observed in DSC pattern.

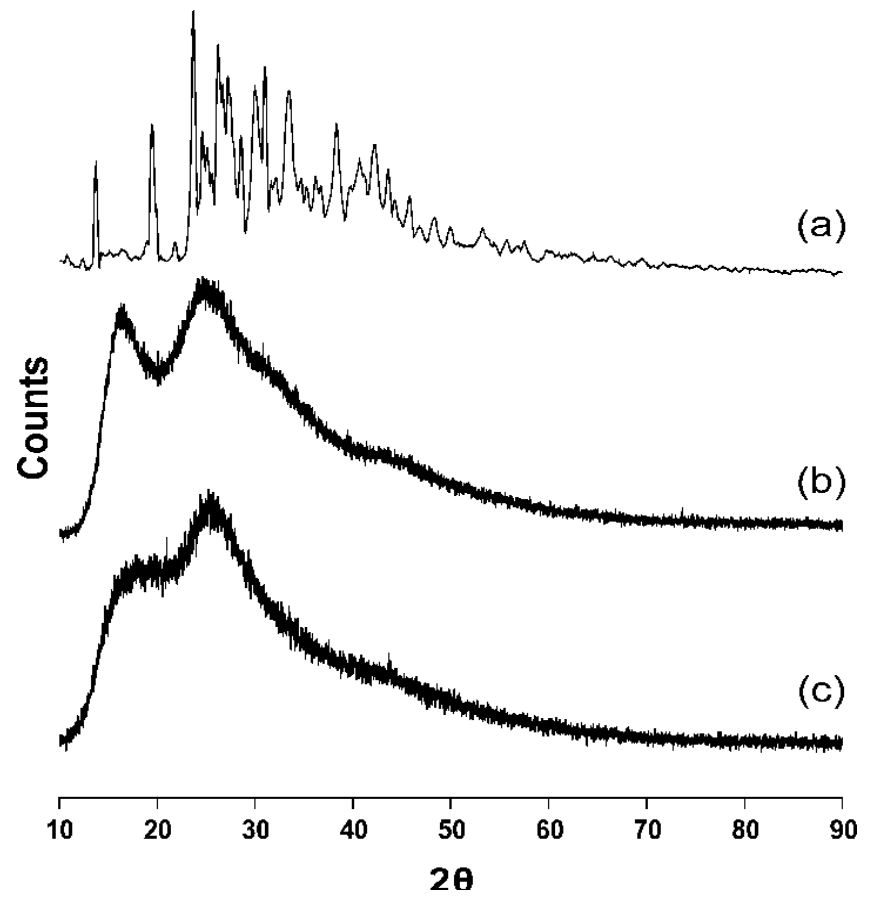

Fig. 7: XRPD patterns of pure cefixime (a), PVP (b) and optimized batch F3 (c).

\section{Fourier transformation infrared spectroscopy (FTIR)}

The possible interaction between drug and polymer was studied by FTIR spectroscopy. The FTIR spectra of cefixime, PVP and an optimized batch of nanoparticles (F3) are shown in Figure 8. The Infrared spectrum of cefixime was characterized by absorption peaks at $2961.71 \mathrm{~cm}^{-1}$ (C-H stretching), $1367.71 \mathrm{~cm}^{-1}$ (N-O stretching), $3523.34 \mathrm{~cm}^{-1}$ (N-H stretching, amine), 1675.81 $\mathrm{cm}^{-1}(\mathrm{C}=\mathrm{O}$ stretching, amide $), 3453.51 \mathrm{~cm}^{-1}$ (O-H stretching, $\mathrm{COOH}$ ), $1326.94 \mathrm{~cm}^{-1}$ (C-N stretching, aromatic), $1770 \mathrm{~cm}^{-1}$ ( $\mathrm{C}=\mathrm{O}$ stretching acid/ester $)$ and $1602.29 \mathrm{~cm}^{-1}(\mathrm{C}=\mathrm{C}$ stretching of an aromatic ring).

The infrared spectrum of PVP showed absorption peaks at $1644.84 \mathrm{~cm}^{-1}$ (C=O stretching), $2952.63 \mathrm{~cm}^{-1}$ (C-H stretching), $3401.37 \mathrm{~cm}^{-1}(\mathrm{O}-\mathrm{H}$ stretching $)$ that were attributed to the presence 
of water, confirming the broad endothermic peak detected in DSC thermo gram (Pandya et al., 2009).

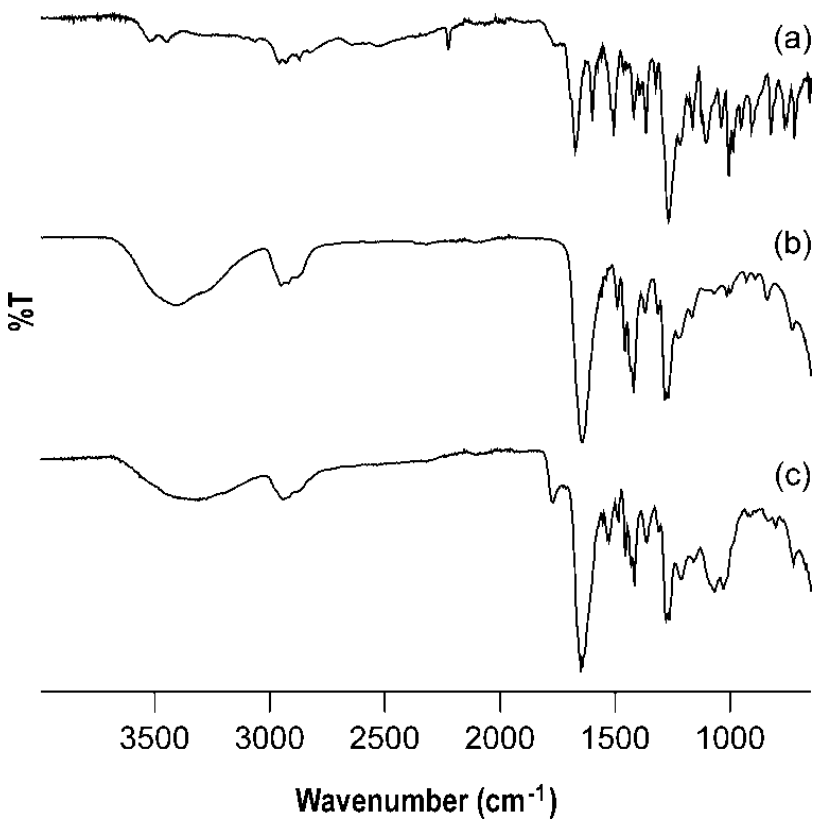

Fig. 8: FTIR spectra of pure cefixime (a), PVP (b) and optimized batch F3 (c)

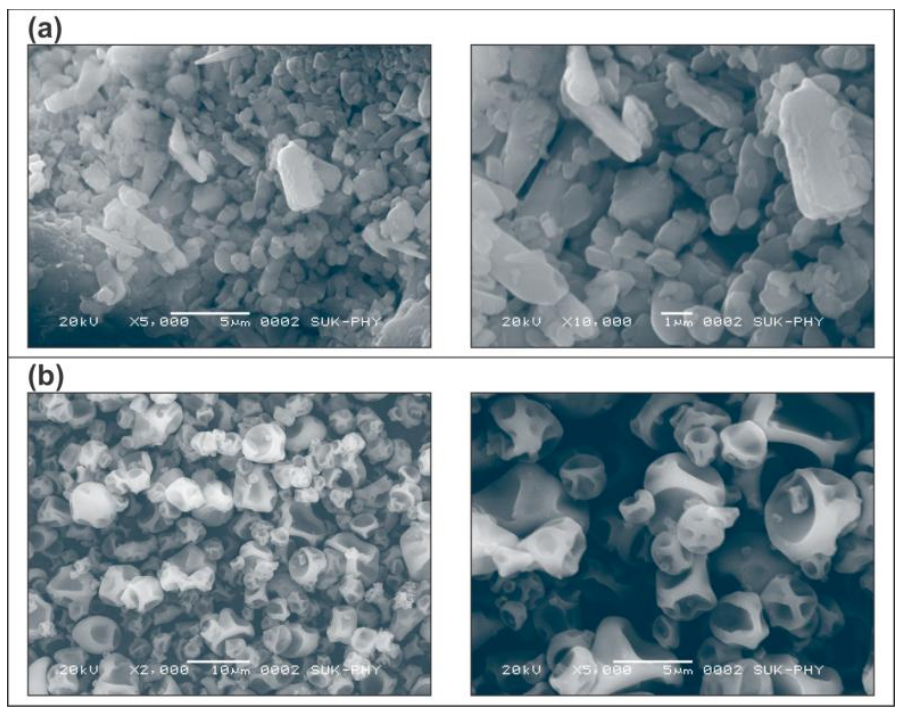

Fig. 9: SEM of pure cefixime (a), and optimized batch F3 (b).

The IR spectrum of an optimized batch of nanoparticles (F3) showed broadening of the peaks of $\mathrm{O}-\mathrm{H}$ stretching vibration at 3400 to $3600 \mathrm{~cm}^{-1}$ due to strong intermolecular hydrogen bonding between cefixime and PVP and all other peaks were found to be smoothened and showed somewhat shifting in the characteristic peaks with decrease in their peak intensities indicating the physical interaction between drug and polymer. An absence of additional peak in an optimized batch of nanoparticles showed that there was no chemical interaction between cefixime and carrier.

\section{Scanning electron microscopy (SEM)}

The surface morphology of the pure cefixime powder, optimized batch of nanoparticles F3 are shown in Figure 9. The SEM micrographs of cefixime powder showed large, irregular, rod shaped particles. Whereas the surface morphology of nanoparticles F3 batch showed hollow and porous spherical particles with smooth or wrinkled (corrugated) surface with uniform and narrow particle size distribution. The SEM image of nanoparticles also showed a little shrinkage occurred in particles, such a type of morphological behavior was found due to the excipient, which led to shrinkage at the particle surface during drying in the chamber and size reduction. From SEM photomicrographs, it could be concluded that the nanoparticles of cefixime exerted a very fine amorphous form with reduction in the particle size, thus remarkably affecting the drug solubility and dissolution rate (Bhosale et al., 2012; Shah et al., 2011).

\section{CONCLUSIONS}

In the present investigation, nanoparticles of poorly water soluble cefixime were successfully prepared by spray drying technique using hydrophilic polymer PVP. Spray drying technique has shown significant reduction of particle size and improved the solubility and dissolution rate of the drug. The characterization of spray dried nanoparticles revealed that the drug was converted into amorphous form. The application of factorial design gave statistically systematic approach for the formulation of nanoparticles with desired particle size, high production yield and percentage drug release within $15 \mathrm{~min}$. The formulation F3 batch was selected as optimized batch with desired properties. The amount of drug: polymer ratio and feed flow rate were found to have significant influence on the particle size, production yield and percentage drug release. Thus, it could be concluded that the use of spray drying technology and hydrophilic carrier to prepare polymeric nanoparticles could improve physicochemical properties of cefixime.

\section{ACKNOWLEDGMENTS}

The corresponding author is grateful to Department of Science and Technology (DST), New Delhi, India for research grant in the form of Fast Track Scheme for Young Scientists (SR/FTP/ETA-53/2011). The authors are thankful to Okasa Pharma, Satara, and Indoco Remedies, Mumbai, Maharashtra, India for providing gift samples of drug and polymer respectively. The analytical facilities provided by Shivaji University, Kolhapur, Poona College of Pharmacy, Pune, and Sinhgad College of Pharmacy, Vadgaon, Pune, Maharashtra, India, are gratefully acknowledged. All the authors express their sincere gratitude toward Principal, Govt. College of Pharmacy, Karad, Maharashtra, India for providing laboratory facilities and constant encouragement.

\section{CONFLICT OF INTERESTS}

The authors report no conflicts of interest. 


\section{REFERENCES}

Alanazi FK, Badry ME, Ahmed MO, Alsarra IA. Improvement of albendazole dissolution by preparing microparticles using spray- drying technique. Sci Pharm, 2007; 75:63-79.

Amidon GL, Lennernas H, Shah VP, Crison JR. A theoretical basis for a biopharmaceutic drug classification: the correlation of in vitro drug product dissolution and in vivo bioavailability. Pharm Res, 1995; 12:413-420.

Bhosale P, Pore Y, Sayyad F. Preparation of amorphous carvedilol polymeric microparticles for improvement of physicochemical properties. J Pharm Investig, 2012; 42:335-344.

Dixit M, Rasheed A, Fijaz NC, Daniel S. Enhancing solubility and dissolution of fenofibrate by spray drying technique. Int $\mathbf{J}$ Pharm Pharm Sci, 2015; 7:173-177.

Hamed E, Sakr A. Application of multiple response optimization technique to extended release formulations design. J Control Release, 2001; 73:329-338

Hecq J, Deleers M, Fanara D, Vranckx H, Amighi K. Preparation and characterization of nanocrystals for solubility and dissolution rate enhancement of nifedipine. Int J Pharm, 2005; 299:167177.

Homayouni A, Sadeghi F, Varshosaz J, Gorekani HA, Nokhodchi A. Compairing various technique to produce micro/ nanoparticles for enhancing the dissolution of celecoxib containing PVP. Eur J Pharm Biopharm, 2014; 88:261-274.

Hussein AA, Mahmood HSh. Preparation and evaluation of cefixime nanocrystals. Iraqi J Pharm Sci, 2014; 23:1-12.

Jadhav P, Petkar B, Pore YV, Kulkarni A, Burade K. Physicochemical and molecular modeling studies of cefixime-L-argininecyclodextrin ternery inclusion compound. Carbohydr Polym 2013; 98:1317-1325

Jamadar S, Pore Y, Sayyad F. Formation of amorphous telmisartan polymeric microparticles for improvement of physicochemical characteristics. Particul Sci Technol, 2014; 32:512-519.

Jondhale S, Bhise S, Pore Y. Physicochemical investigations and stability studies of amorphous gliclazide. AAPS PharmSciTech, 2012; 13:448-459.

Kawabata Y, Wada K, Nakatani M, Yamada S, Onoue S. Formulation design for poorly water-soluble drugs based on biopharmaceutics classification system: basic approaches and practical applications. Int J Pharm, 2011; 420:1-10.

Khadka P, Ro J, Kim H, Kim I, Kim JT, Kim H, Cho JM, Yun G, Lee J. Pharmaceutical particle technologies: An approach to improve drug solubility, dissolution and bioavailability. Asian J Pharm Sci, 2014; 9:304-316.

Kornblum SS, Hirschorn JO. Dissolution of poorly water soluble drugs. I. Some physical parameters related to method of micronization and tablet manufacture of quinazolinone compound. J Pharm Sci, 1971; 59:606-609.

Kornblum SS, Hirschorn JO. Dissolution of poorly water soluble drugs. II. Excipient dilution and force of compression effects on tableting of quinazolinone compound. J Pharm Sci, 1971; 60:445-448.

Kristmundsdottir $\mathrm{T}$, Gundmundsson OS, Ingvarsdottir $\mathrm{K}$. Release of diltiazem from eudragit microparticles prepared by spraydrying. Int J Pharm, 1996; 137:159-165.

Lepek P, Sawicki K, Wlodaraski Z, Wojnarowska M, Paluch, Guzik L. Effect of amorphization method of telmisartan solubility and the tableting process. Eur J Pharm Biopharm, 2013; 83:114-121.

Motlekar N, Youan B. Optimization of experimental parameters for the production of LMWH- loaded polymeric microspheres. Drug Des Dev Ther, 2008; 2:39-47.

Murtaza G, Ahmad M, Khan SA, Hussain I. Evaluation of cefixime-loaded chitosan microspheres: analysis of dissolution data using DDSolver. Dissolut Technol, 2012; 19:13-19.
Nagda C, Chotai NP, Patel U, Parel S. Preparation and characterization of spray dried mucoadhesive microspheres of aceclofenac. Drug Dev Ind Pharm, 2009; 35:1155-1166.

Noyes AA, Whitney WR. The rate of solution of solid substances in their own solutions. J Am Chem Soc, 1897; 19:930-934.

Padamwar MN, Pokharkar VB. Development of vitamin loaded topical liposomal formulation using factorial design approach: drug deposition and stability. Int J Pharm, 2006; 320:37-44.

Pandya VM, Patel DJ, Patel JK, Patel RP. Formulation, characterization, and optimization of fast dissolve tablets containing celecoxib solid dispersion. Dissolut Technol, 2009; 441:424-432.

Proudfoot S. 1991. Factors affecting bioavailability: factors influencing drug absorption from gastrointestinal tract. In: Aulton ME, ed. Pharmaceutics: The Science of Dosage Form Design. Edinburgh: Churchil Livingstone 135-73.

Rao S, Song Y, Peddie F, Evans AM. Particle size reduction to the nanometer range: a promising approach to improve buccal absorption of poorly water-soluble drugs. Int J Nanomedicine, 2011; 6:1245-1251.

Raval JP, Naik DR, Patel PS. Spray-dried cefixime encapsulated poly(lactide-co-glycolide) microparticles: characterization and evaluation of in vitrorelease kinetics with antibacterial activity. Drying Technol, 2012; 30:865-872.

Rhee YS, Chang SY, Park CW, Chi SC, Park ES. Optimization of ibuprofen gel formulations using experimental design technique for enhanced transdermal penetration. Int J Pharm, 2008; 364:14-20.

Roni AM, Dipu HM, Kibria G, Rahman H, Rony RM, Jalil RU. Dissolution enhancement of poorly soluble carbamazepine by using polymeric solid dispersions. Int J Pharm Sci Res, 2011; 2:49-57.

Sahoo NG, Kakran M, Li L, Jugeh Z, Muller RH. Dissolution enhancement of a poorly water- soluble antimalarial drug by means of modified multi-fluid nozzle pilot spray drier. Mater Sci Eng C, 2011 31:391-399.

Shah N, Kondawar M, Shah R, Shah V. Sustained release of spray dried combination dry powder inhaler formulation for pulmonary delivery. Asian J Pharm Clin Res, 2011; 4:112-118.

Shinde ML, Shah VA, Ghodke DS, Shah RR. Impact of formulation and process variable on the preparation of acyclovir microsphere by spray drying using factorial design. Int J Res Pharm Sci, 2010; 1:353-357.

Singh D, Harikumar SL, Nirmala. Nanoparticles: An Overview. J Drug Deliv Ther, 2013; 3:169-175.

Sonam, Chaudhary H, Kumar V. Taguchi design for optimization and development of antibacterial drug-loaded PLGA nanoparticles. Int J Biol Macromol, 2014; 64:99-105.

Tran T, Poudel HBK, Marasini N, Chi SC, Choi HG, Yong CS, Kim JO. Preparation and evaluation of raloxifene loaded solid dispersion nanoparticles by spray drying technique without an organic solvent. Int J Pharm, 2013; 443:50-57.

Wan L, Hang P, Chia C. Preparation of coated particles using spray drying process with an aqueous system. Int J Pharm, 1991; 77:183191.

Yadav HKS, Nagavarma BVN, Ayaz A, Vasudha LS, Shivakumar HG. Different techniques for preparation of polymeric nanoparticles: A Review. Asian J Pharm Clin Res, 2012; 5:16-23.

\section{How to cite this article:}

Desai PS, Pore YV. Physicochemical characterization of spray dried cefixime polymeric nanoparticles using factorial design approach. J App Pharm Sci, 2016; 6 (04): 124-132. 